\title{
Was erschwert die Aufdeckung organisierter und ritueller Gewaltstrukturen?
}

\author{
Eine qualitative Inhaltsanalyse der Erlebnisberichte von Betroffenen und \\ Zeitzeug_innen
}

\section{Which Factors Impede the Uncovering of Organized and Ritual Child Sexual Abuse?}

\section{A Qualitative Content Analysis of Reports of Victims and Contemporary Witnesses}

\section{(C) $(1)$}

Autoren

Johanna Schröder ${ }^{1}$, Pia Behrendt ${ }^{1}$, Susanne Nick ${ }^{1,2}$, Peer Briken ${ }^{1}$

Institute

1 Institut für Sexualforschung, Sexualmedizin und Forensische Psychiatrie, Universitätsklinikum HamburgEppendorf

2 Klinik und Poliklinik für Psychiatrie und Psychotherapie, Universitätsklinikum Hamburg-Eppendorf

\section{Schlüsselwörter}

sexueller Kindesmissbrauch, dissoziative Identitätsstörung, posttraumatische Belastungsstörung, organisierte Gewalt, rituelle Gewalt

Keywords

child sexual abuse, dissociative identity disorder, posttraumatic stress disorder, organized abuse, ritual abuse

\section{Bibliografie}

DOI https://doi.org/10.1055/a-1123-3064

Online-Publikation: 8.4.2020 | Psychiat Prax 2020; 47:

249-259

(c) Georg Thieme Verlag KG Stuttgart · New York

ISSN 0303-4259

\section{Korrespondenzadresse}

Dr. phil. Johanna Schröder, Institut für Sexualforschung, Sexualmedizin und Forensische Psychiatrie, Universitätsklinikum Hamburg-Eppendorf, Martinistraße 52,

20246 Hamburg

jo.schroeder@uke.de

\section{ZUSAMMENFASSUNG}

Ziel der Studie Untersuchung sexuellen Kindesmissbrauchs in organisierten und rituellen Gewaltstrukturen (d. h. vernetzte Täter_innengruppen mit ideologischen Hintergründen).
Methodik Es wurden 33 Berichte von Betroffenen und Zeitzeug_innen mittels qualitativer Inhaltsanalyse ausgewertet. Dabei wurden in Bezug auf die Fragestellung, welche Faktoren die Aufdeckung solcher Strukturen erschweren, induktiv Kategorien gebildet.

Ergebnisse Die Ergebnisse legen nahe, dass verschiedene (Beziehungs-)Aspekte dreier wechselseitig interagierender Personengruppen die Aufdeckung solcher Strukturen erschweren können: Täter_innen, die Betroffenen und andere Personen (z. B. im direkten Umfeld der Betroffenen und in der Gesellschaft).

Schlussfolgerung Um die beschriebenen Systemfaktoren im Sinne einer verbesserten Aufdeckung dieser Gewaltstrukturen positiv beeinflussen zu können, ist eine sachliche Wissensvermittlung an verschiedene Institutionen der Gesellschaft notwendig.

\section{ABSTRACT}

Objective The study aims to investigate organized and ritual child sexual abuse (i.e. networking perpetrator groups with ideological frameworks).

Methods A qualitative content analysis was conducted on 33 reports by victims and contemporary witnesses. Inductive categories were formed with regard to the question, which factors might impede the exposure of such structures.

Results The results suggest that several interdependent factors of three interacting groups may play major roles in the deficient detection of those structures: the perpetrators, the survivors and other persons (e. g. in the vicinity of the survivors and in society).

Conclusion In order to influence the described system of interdependent factors positively in the sense of an improved exposure of those violent structures, the objective communication of knowledge in different societal institutions is necessary. 


\section{Einleitung}

Im Jahr 2016 berief der Unabhängige Beauftragte für Fragen des sexuellen Kindesmissbrauchs (UBSKM) auf Grundlage des Beschlusses des Deutschen Bundestags die Unabhängige Kommission zur Aufarbeitung sexuellen Kindesmissbrauchs (UKASK) mit dem Auftrag ein, Ursachen, Bedingungen und Folgen von sexuellem Kindesmissbrauch in Deutschland zu untersuchen. Darüber hinaus ist ein Ziel der UKASK, die Gesellschaft gemeinsam mit Betroffenen über das Ausmaß von sexuellem Kindesmissbrauch zu informieren und für das Thema zu sensibilisieren, da deren Erfahrungsberichte für die Aufarbeitung eine zentrale Rolle einnehmen [1].

Im ersten Bilanzbericht der UKASK zeigte sich, dass etwa 10\% $(n=117)$ der bis dahin ausgewerteten 914 (von bis dahin 1216 eingegangenen) Anhörungen und Berichte sexuellen Kindesmissbrauch in organisierten Strukturen thematisierten [1]. Organisierte Gewaltstrukturen sind insbesondere durch eine systematische Anwendung schwerer sexualisierter, körperlicher und psychischer Gewalt an Kindern, Jugendlichen und Erwachsenen gekennzeichnet, werden durch eine Zusammenarbeit mehrerer Täter_innen bzw. Netzwerke ermöglicht und sind häufig mit kommerzieller sexueller Ausbeutung (z. B. Zwangsprostitution, Menschenhandel und sogenannter Kinder-/Gewaltpornografie) verbunden [2, 3]. Ein Großteil von 165 Betroffenen aus Deutschland, die im Rahmen einer anonymen Onlinestudie befragt wurden, berichteten, über die eigene Familie in das organisierte Netzwerk gelangt zu sein [4] und dass bereits die Elterngeneration darin aufgewachsen sei [5]. Von den 117 Anhörungen und Berichten aus dem Kontext organisierter Gewalt wurden in 42 Fällen sogenannte „rituelle“ Strukturen deutlich [1], die sich durch (Schein-)Ideologien auszeichnen, welche zur Sinngebung, Rechtfertigung und Intensivierung von Gewalt, zur Bindung von Personen an die Täter_innen-Gruppe und zum Machterhalt der Täter_innen dienen können [1, 3, 6]. Häufige Beispiele hierfür sind satanistische und rechtsextreme Gruppierungen sowie religiöse/freikirchliche Sekten und Kulte $[7,8]$, die oft patriarchal-hierarchisch strukturiert sind $[1,6]$. Im Kontext von organisierter und ritueller Gewalt (ORG) berichten viele Betroffene von verschiedenen Formen der Bewusstseinsmanipulation, insbesondere von der strategischen Erzeugung einer dissoziativen Identitätsstörung (DIS; früher „multiple Persönlichkeitsstörung“) [1, 9]. Eine DIS kann durch extreme Gewaltanwendungen im frühen Kindesalter entstehen und ist durch eine Desintegration des Bewusstseins in Bezug auf die eigene Identität sowie auf Affekte, Kognitionen und das Verhalten gekennzeichnet [10]. Dieser teilweise amnestische dissoziative Mechanismus [11] ermöglicht Menschen, anhaltende traumatische Erlebnisse aus dem Bewusstsein auszugrenzen und dient so dem Erhalt der Funktionsfähigkeit im Alltag [3], wozu auch das Bewahren der als überlebenswichtig empfundenen Beziehung zu den misshandelnden Eltern gehören kann [12]. Die mit einer DIS einhergehenden Teil-Persönlichkeiten können nach Angaben von Betroffenen ideologisch indoktriniert (sog. mind control) und unter Anwendung massiver Gewalt, psychotroper Substanzen, sensorischer Deprivation oder Täuschung trainiert werden, um mithilfe der Auslösung konditionierter Reize (sog. Programmierung) Anweisungen der Täter_innen (z. B. Suizid) oder Regeln der Gruppe (z. B. Schweigegebote) zu befolgen [3].

Die dissoziative Abspaltung der Gewalterfahrungen ermöglicht vielen Betroffenen ein Durchhalten und Überleben im traumaerzeugenden Umfeld, wobei eine DIS (z. B. durch die damit verbundenen Erinnerungslücken und Kontrollverluste) langfristig oft mit einer starken psychischen Belastung einhergeht $[1,13]$. Darüber hinaus entwickeln Personen aus ORGStrukturen oft weitere Traumafolgestörungen [4, 14, 15], wie komplexe posttraumatische Belastungsstörungen und somatoforme Dissoziationssymptome, die in rituellen noch stärker auftreten als in organisierten Gewaltkontexten [4]. Ein Ausstieg aus dem Gewaltumfeld wirkt sich positiv auf die psychische Symptombelastung aus [4], wobei viele Betroffene einen Mangel an professioneller Unterstützung bei Ausstiegsversuchen und in der medizinischen/psychotherapeutischen Versorgung benennen $[5,16]$. Diese Versorgungslücke beruht, nach der Einschätzung der UKASK und einer Befragung unter deutschen Fachkräften mit Kontakt zu Kindern und Jugendlichen, unter anderem auf einer ausgeprägten Skepsis aufseiten psychosozialer Fachpersonen [1, 17].

Die sogenannte "Glaubensfrage“ begleitet das Thema ORG schon lange [1] und äußert sich unter anderem darin, dass Kritiker_innen Erfahrungsberichte als (z. B. durch Therapeut_innen suggerierte) Pseudoerinnerungen dementieren [18]. Nach einer Stellungnahme des Betroffenenrates des UBSKM zum Umgang mit ritueller Gewalt existiert seit 20 Jahren ein Narrativ zu ritueller Gewalt, das von Unglaube geprägt ist und so eine wissenschaftliche Beschäftigung mit dieser Gewaltform verzögert sowie adäquate Hilfen erschwert [19]. Die vorliegende Studie verfolgt das Ziel, an diesem Narrativ zu arbeiten und Aufklärungsarbeit zu leisten. Hierfür ist es wichtig zu ergründen, warum Strukturen organisierter und ritueller Gewalt weitgehend verborgen bleiben und welche Faktoren aus Sicht der betroffenen Personen dazu beitragen.

\section{Methoden}

\section{Studiendesign und -material}

Betroffene von sexuellem Missbrauch in der Kindheit und Jugend wurden von der UKASK dazu eingeladen, ihre Erfahrungen in vertraulichen Anhörungen und schriftlichen Berichten zu schildern. Die Anhörungen fanden in einem geschützten Rahmen statt, wurden bundesweit von UKASK-Mitgliedern und Anhörungsbeauftragten durchgeführt und dienten nicht primär zu Forschungszwecken. Für die Anhörungen und Berichte wurden einige nicht obligatorische Themen zur Orientierung vorgegeben (z.B. Art und Kontext der Missbrauchserfahrungen und Tatfolgen). Alle Angaben, die einen Rückschluss auf beteiligte Personen oder Orte erlaubten, wurden von Mitarbeiter_innen der UKASK in den Berichten sowie in den Transkripten der Anhörungen zensiert und nach informierter Einwilligung der Berichtenden den Autor_innen doppelt pseudonymisiert zur Analyse zur Verfügung gestellt. Das Studiendesign wurde von der Ethikkommission der Psychotherapeutenkammer Hamburg geprüft. Aufgrund der beschriebenen Rahmenbedingungen 
entspricht die Auswahlmethode des Materials der „anfallenden Stichprobe“, also einer Aufnahme nach Verfügbarkeit [20]. Alle im Zeitraum von Mai 2016 bis November 2018 angefallenen Dokumente, die im ersten Schritt von den Mitarbeiter_innen der UKASK und im zweiten Schritt von den Autor_innen dem Themenbereich ORG zugeordnet wurden, erfüllten die Kriterien für einen Einschluss in die Datenanalyse.

\section{Datenanalyse}

Die Daten wurden mit der Software ATLAS.ti [21] im Sinne einer inhaltlich-strukturierenden Qualitativen Inhaltsanalyse nach Mayring ausgewertet, deren Ziel es ist, bestimmte Themenaspekte aus dem Material herauszufiltern [22]. Mangels einer starken zugrunde liegenden Theorie über vorhandene Kategorien wurde bei der Kategorienbildung, entsprechend der „konventionellen Kategorienentwicklung“ nach Hsieh u. Shannon [23] ein induktives Vorgehen gewählt. In einem ersten Durchlauf wurden bei der Bearbeitung des ersten Drittels des Materials für die Fragestellung relevant erscheinende Textstellen markiert, bei der Bearbeitung des zweiten Drittels ein vorläufiges Kategoriensystem definiert und im letzten Drittel wurde das vorläufige Kategoriensystem erprobt und modifiziert (z. B. Kategorien überprüft, abstrahiert und reduziert). In einem zweiten Durchlauf fand eine Kategorisierung anhand des finalen Kategoriensystems statt. Im Anschluss erfolgte eine Überprüfung der Interraterreliabilität durch 3 unabhängige Rater anhand eines zufällig ausgewählten Dokuments. Die Berechnung wurde mit dem ,irr' package [24] in der Software $R$ [25] durchgeführt.

\section{Ergebnisse}

\section{Stichprobenbeschreibung}

Es wurden 42 der bei der UKASK eingegangenen Fälle dem Kontext ORG zugeordnet. Davon gingen 33 Fälle in die vorliegende Analyse ein, darunter doppelt so viele transkribierte Anhörungen wie schriftliche Berichte ( $\triangleright$ Tab. 1). Ausgeschlossen wurden 9 Dokumente (z. B. wegen Rückzug der Einwilligung oder abgebrochenen Interviews). Die Befragten waren durchschnittlich 46 Jahre alt, zum Großteil weiblich und beschrieben in den meisten Fällen eigene Erfahrungen in ORG-Kontexten (Primärquellen). Die wenigen Sekundärquellen waren psychosoziale Fachpersonen, die von ORG Betroffene professionell begleitet haben. In fast allen Fällen gehörten Personen aus dem Familienkreis der betroffenen Personen zu der Täter_innengruppe. Oft wurde von einem Ausstieg aus der Täter_innengruppe und von (erzwungener) Mittäterschaft berichtet. Außerdem zeigten sich oft Hinweise auf eine DIS, wobei der Anteil an Fällen mit unklaren Angaben im Material vergleichsweise groß war. Bei 12 der 33 Berichtenden wurde nach der Inhaltsanalyse die Einwilligung zur Veröffentlichung bestimmter Zitate erfragt, wovon 10 Personen zustimmten.

\section{Struktur des Kategoriensystems}

In der Datenanalyse bildeten sich 3 Oberkategorien heraus, in denen sich die involvierten Personengruppen widerspiegeln, die systemisch miteinander interagieren. Innerhalb der Oberka-
- Tab. 1 Charakteristika der von organisierter und ritueller Gewalt berichtenden Betroffenen und Zeitzeug_innen $(N=33)$.

analysiertes Material

\begin{tabular}{|l|r|r|}
\hline Anhörung/Interview $(n, \%)$ & 22 & 66,7 \\
\hline schriftlicher Bericht $(n, \%)$ & 10 & 30,3 \\
\hline beides & 1 & 3,0 \\
\hline
\end{tabular}

Quelle des Berichts

\begin{tabular}{|l|r|r|}
\hline primär $(n, \%)$ & 27 & 81,8 \\
\hline sekundär $(n, \%)$ & 4 & 12,1 \\
\hline primär \& sekundär $(n, \%)$ & 2 & 6,1 \\
\hline
\end{tabular}

Geschlecht der Berichtenden

\begin{tabular}{|l|r|r|}
\hline männlich $(n, \%)$ & 3 & 9,1 \\
\hline weiblich $(n, \%)$ & 27 & 81,8 \\
\hline k. A. $(n, \%)$ & 3 & 9,1 \\
\hline
\end{tabular}

Alter in Jahren

\begin{tabular}{|l|c|c|}
\hline$M(S D)$ & 46,3 & $(10,8)$ \\
\hline k. A. $(n, \%)$ & 9 & 27,3 \\
\hline
\end{tabular}

Familie in Täter_innengruppe involviert

\begin{tabular}{|l|r|c|}
\hline ja $(n, \%)$ & 32 & 97,0 \\
\hline nein $(n, \%)$ & 0 & 0 \\
\hline k. A. $(n, \%)$ & 1 & 3,0 \\
\hline
\end{tabular}

Bericht von Ausstieg

\begin{tabular}{|l|c|c|}
\hline gelungen $(n, \%)$ & 21 & 63,6 \\
\hline teilweise gelungen $(n, \%)$ & 4 & 12,1 \\
\hline nicht gelungen $(n, \%)$ & 0 & 0 \\
\hline k. A. $(n, \%)$ & 8 & 24,2 \\
\hline
\end{tabular}

Bericht von oder Hinweis auf DIS

\begin{tabular}{|c|r|r|}
\hline ja $(n, \%)$ & 24 & 72,7 \\
\hline nein $(n, \%)$ & 5 & 15,2 \\
\hline k. A. $(n, \%)$ & 4 & 12,1 \\
\hline Bericht von Mittäterschaft & & \\
\hline
\end{tabular}

\begin{tabular}{|l|r|r|}
\hline ja $(n, \%)$ & 7 & 21,2 \\
\hline nein $(n, \%)$ & 3 & 9,1 \\
\hline k. A. $(n, \%)$ & 23 & 69,7 \\
\hline
\end{tabular}

Anmerkungen: $k$. A. = keine genaue Angabe; $M=$ Mittelwert, $S D=$ Standardabweichung; DIS = Dissoziative Identitätsstörung

tegorien wurden verschiedene Unterkategorien deutlich, in denen sich Faktoren ausdrücken, die zur erschwerten Aufdeckung von organisierter und ritueller Gewalt beitragen. Die Unterkategorien wurden teilweise durch spezifische Facetten weiter ausdifferenziert. In der Ergebnisdarstellung werden die Unterkategorien im Kontext der 3 Oberkategorien systematisch beschrieben und mit Beispielzitaten belegt. Die Interraterreliabili- 
Tab. 2 Oberkategorie I: Täter_innen.

\begin{tabular}{|c|c|c|}
\hline $\begin{array}{l}\text { Faktoren, die die Aufdeckung von organi- } \\
\text { sierter und ritueller Gewalt erschweren } \\
\text { (Unterkategorien a-e) }\end{array}$ & $\Sigma$ & \\
\hline a) Gewalt & & 220 \\
\hline psychische Gewalt & 122 & \\
\hline \multicolumn{3}{|l|}{ Bedrohung (53) } \\
\hline \multicolumn{3}{|l|}{ Überwachung (44) } \\
\hline \multicolumn{3}{|l|}{ Erpressung (25) } \\
\hline körperliche Gewalt & 64 & \\
\hline \multicolumn{3}{|l|}{ psychoaktive Substanzen (23) } \\
\hline \multicolumn{3}{|l|}{ Folter (15) } \\
\hline \multicolumn{3}{|l|}{ Tötungen (26) } \\
\hline sexualisierte Gewalt & 34 & \\
\hline \multicolumn{3}{|l|}{ Vergewaltigung (11) } \\
\hline \multicolumn{3}{|l|}{ Zwangsprostitution (21) } \\
\hline \multicolumn{3}{|l|}{ Kinderpornografie (2) } \\
\hline b) gesellschaftliche Positionierung & & 141 \\
\hline sozial angepasste Erscheinung & 20 & \\
\hline finanzielle Ressourcen & 25 & \\
\hline Bekleidung von Machtpositionen & 53 & \\
\hline Organisation/Vernetzung & 43 & \\
\hline c) Manipulation & & 92 \\
\hline Induzieren/Nutzen von DIS & 16 & \\
\hline Programmierung/Mind control & 39 & \\
\hline Gaslighting & 7 & \\
\hline $\begin{array}{l}\text { Ausnutzung psychologischer Grund- } \\
\text { bedürfnisse }\end{array}$ & 6 & \\
\hline Indoktrination/(Pseudo-)Ideologien & 24 & \\
\hline d) Vertuschung & & 84 \\
\hline Schweigegebote & 33 & \\
\hline $\begin{array}{l}\text { Verschleierung von Identitäten/ } \\
\text { Tatorten }\end{array}$ & 8 & \\
\hline Abstreiten bei Konfrontation & 14 & \\
\hline Vertuschen von körperlichen Gewaltspuren & 22 & \\
\hline Diskreditierung von Opfern & 5 & \\
\hline Sabotage polizeilicher Ermittlungen & 2 & \\
\hline e) Täter_innen-loyales Familiensystem & & 14 \\
\hline Familiensystem isoliert sich & 3 & \\
\hline Familiensystem schützt sich & 11 & \\
\hline Anmerkungen: DIS: Dissoziative Identitätsstörung & & \\
\hline
\end{tabular}

\section{Faktoren, die die Aufdeckung von organi- sierter und ritueller Gewalt erschweren gorien a-e)}

a) Gewalt tät für die Ober- und Unterkategorien lag bei $\kappa=0,63$, was einer substanziellen Übereinstimmung entspricht.

\section{Oberkategorie I: Täter_innen}

In > Tab. 2 werden Unterkategorien dargestellt, in denen sich durch Täter_innen bewirkte Faktoren ausdrücken, die zur Verschleierung von ORG-Strukturen beitragen.

\section{a) Täter_innen und Gewalt}

Es wird berichtet, dass die Täter_innen durch psychische Gewalt in Form von Androhung körperlicher Gewalt, Verfolgung und Erpressung (z.B. mit Bildmaterial von erzwungener Mittäterschaft) Kontrolle über die Betroffenen ausübten. Durch die Erpressung von Fremdtäter_innen im Kontext von Zwangsprostitution (z.B. mit Bildmaterial von sexualisierter Gewalt) sei ihnen auch das Schweigen dieser involvierten Personengruppe gesichert.

„Ich musste Kinder auf diese Gewalttaten vorbereiten. [...] Und um den anderen beizubringen, wie sie überleben können, musste ich denen das auch gut beibringen. [...] Mit der Schuld bindet der Kult einen ja an den Kult."

„Man verfolgte mich und man setzte mich auch unter Druck, indem man mir meine eigenen Straftaten vorgehalten hat."

„Aufgrund der Erpressbarkeit können solche Menschen dazu gebracht werden, Dinge zu tun, die letztlich dem Schutz des Kultes dienen."

Die Anwendung von körperlicher Gewalt durch Täter_innen könne sowohl sadistisch als auch strategisch motiviert sein (z. B. zu kommerziellen Zwecken). Berichtet werden beispielsweise die Applikation von sedierenden Substanzen und diverse Foltermethoden, bis hin zu Tötungen.

„Nicht nur im Familienleben ergötzte er sich an der Ausübung von Macht in Verbindung mit Gewalt. “

„In dem Kult bezahlen die ganz viel Geld für Folterungen von Kindern, da zuzugucken, mitzumachen.“

Sexualisierte Gewalt durch die Täter_innen wird ebenfalls als sadistisch oder strategisch motiviert beschrieben (z. B. Vergewaltigungen vs. kommerzielle Zwangsprostitution).

„Vor allem war er aber offenbar sexuell nur erregbar, wenn er seine Macht- und Gewaltphantasien ausleben konnte.“

„Und dann kamen Leute, Männer. Die haben sich ein Kind gemietet und dafür bezahlt. Also das war ein Umschlagplatz für Kinderprostitution.“

\section{b) Täter_innen und ihre gesellschaftliche Positionierung}

Die Berichtenden beschreiben die Täter_innen als sozial angepasst und unauffällig bis hin zu gesellschaftlich angesehen. Weiterhin wird dargestellt, dass die Täter_innen oft in gesellschaftlich angesehenen Berufen arbeiten (z. B. kirchliche Ämter, Kommunalpolitiker_innen, Ärzt_innen, Apotheker_innen, Polizist_innen, Richter_innen oder Rechtsanwält_innen). Diese sind den Schilderungen zufolge gut organisiert und vernetzt (z. B. in kooperierenden, teilweise hierarchisch organisierten Gruppen) und bekleiden teilweise politisch, wirtschaftlich oder gesellschaftlich einflussreiche Positionen. Es wird beschrieben, dass diese Machtpositionen teils durch finanzielle Ressourcen gesichert würden, die im Rahmen von Zwangsprostitution und 
der Erstellung sogenannter Kinderpornografie entstünden (siehe Kategorie $l-a$ ).

„Für Deutschland strebt die Führungsebene des Kultes an, hohe Posten in allen wichtigen Bereichen (Politik, Wirtschaft, Justiz, Polizei, Kirche) mit loyalen mit ,ideologisch reinen 'Mitgliedern zu besetzen. Die Täter setzen all ihr Wissen, ihre Verbindungen und viel Geld ein, um eine Aufdeckung zu verhindern. Naturgemäß haben demnach Sadismus liebende und gleichzeitig kluge Menschen die größten Chancen, sowohl in wichtige Führungsebenen des Kultes als auch an Machtpositionen in der Außenwelt zu gelangen. “

„Die Täter sind nicht irgendwelche armen Schlucker, sondern das ist der Staatsanwalt, das ist Bischof, das ist der Richter. Das sind Menschen, die Ansehen und Geld haben. Also das ist auch wichtig, dass wir in allen Schichten die Täter suchen. “

\section{c) Täter_innen und Manipulation}

Die Berichtenden schildern eine strategische Nutzung verschiedener psychischer Manipulationstechniken durch die Täter_innen. Besonders bedeutsam sei in diesem Kontext die Indoktrination im Sinne einer Manipulation durch (Pseudo-)Ideologien. Dies seien (z.B. satanische, religiöse, spirituelle oder rechtsextreme) Glaubensgrundsätze, die von den Täter_innen entweder selbst angenommen oder strategisch genutzt würden, um Menschen an die Gruppe (z. B. eine Sekte) zu binden, Gewalttaten zu legitimieren und Widerstand zu hemmen. Oft wird beschrieben, dass die Täter_innen den Betroffenen vermitteln, sie gehörten im Vergleich zu „unwürdigen“ Menschen außerhalb des Kultes zur Elite der Gesellschaft.

„Die Strafe ist ja sozusagen das Tolle, weil wir dadurch halt dem Teufel näherkommen. Also je mehr Strafe, je mehr Folter, je mehr Vergewaltigung, je mehr Elektroschocks, je mehr Schmerz, desto größer unsere Chance, dahin zu kommen. Das ist das Auserwählte. Das ist paradox aus heutiger Sicht, aber das ist das Denken von denen gewesen. Also wir müssen uns glücklich schätzen, dass wir vergewaltigt wurden und dass wir gefoltert wurden und so."

Weiterhin wird emotionale Vernachlässigung und die Ausnutzung psychologischer Grundbedürfnisse (z. B. Bindung und Selbstwert) geschildert, ebenfalls mit dem Ziel, das Zugehörigkeitsgefühl der Betroffenen zur Gruppe zu stärken.

„Und auch die Geborgenheit, irgendwo einer Gruppe dazuzugehören, weil man sagte mir immer, dass man etwas Besonderes ist, wenn man dieser Gruppierung angehört. “

„Bei einer Vergewaltigung hat er gesagt: Ich liebe dich. Und man ankert sich daran. Also ich liebe dich. Man braucht ja Liebe. Und er vergewaltigt einen dabei."

Es wird auch berichtet, dass durch massive Traumatisierungen im frühen Kindesalter der betroffenen Personen dissoziative Identitätsstörungen erzeugten und die hierdurch entstandenen abgespaltenen Persönlichkeitsanteile von den Täter_innen nutzbar gemacht würden.

"Soweit ich weiß, hat mein Erzeuger mich quasi von Geburt an auf das Kultleben vorbereitet. Dabei nutzte er offenbar vorhandenes Kultwissen, durch extreme Schmerzen und Todesnähe, dissoziierte Persönlichkeitsanteile zu, erschaffen'. Diese ,Erziehung' begann wahrscheinlich zunächst zu Hause oder lief parallel zum Kultleben ab. Erste gezielt ,erzeugte‘ Persönlichkeitsanteile sind bereits im Säuglingsalter entstanden. “
Die Zweckdienlichkeit der Persönlichkeitsanteile Betroffener bestehe in der psychischen Konditionierung (bzw. der Festlegung und Automatisierung) der von den Täter_innen gewünschten Verhaltensweisen, die den Berichten zufolge an bestimmte dissoziative Identitäten gebunden ist und durch konditionierte sensorische Reize ausgelöst werden kann (sog. Programmierung/mind control), z. B. im Sinne einer Verhaltenslenkung der Betroffenen durch Schlüsselwörter zum Einsatz in der Zwangsprostitution.

„Wenn ich versuche, Sachen von damals zu rekonstruieren, kann es sein, dass Programme anspringen, die mich dazu bringen, irgendwohin zu gehen oder irgendwas zu tun. [...] Ein Programm ist ja nichts anderes als ein Training, ein gutes Training. So wie Sie einem Kind beibringen, an der roten Ampel stehenzubleiben, können Sie einem Kind auch beibringen, auf ein Fingerschnippen hin sich auszuziehen. Das ist nicht schwer."

Weiterhin wird von sogenanntem „Gaslighting“ berichtet, worunter eine gezielte Manipulation des Realitätsbewusstseins verstanden wird, indem Täter_innen dauerhaft und wiederholt die Realitätswahrnehmung der betroffenen Personen infrage stellen. Ein Beispiel hierfür ist die Verleugnung oder die bewusste Inszenierung von Ereignissen.

„Um mir die Schuld zu geben, werden dann Filme gezeigt. Anteile, die mehr Bewusstsein haben, die sehen mich als Person da stehen, wie ich ein Kind foltere. Dass aber in dieser Szene fünf Persönlichkeiten zum Beispiel daran beteiligt sind, die immer nur ein bisschen wissen, das sehe ich ja von außen nicht. Ich als diejenige, die den Film dann sieht, sehe nur, dass ich da handele und fühle mich schuldig. “

\section{d) Täter_innen und Vertuschung}

Nach Angabe der Berichtenden setzen Täter_innen vielfältige Strategien ein, um Gewalttaten an Betroffenen nicht erkennbar werden zu lassen. Hierzu zählen Schweigegebote (z. B. durch Bedrohung, Erpressung und Bestrafung; siehe Kategorie I-a), die Nutzung versteckter Tatorte, das Verheimlichen von Täter_innen-Identitäten (z. B. keine Nennung von Namen, Maskierungen), das Abstreiten von Gewalttaten (z.B. bei Konfrontation durch das Jugendamt oder Lehrer_innen), das Vertuschen von Spuren körperlicher Gewalteinwirkungen bei den Betroffenen (z. B. durch Täter_innen-assoziierte oder erpressbare Ärzte/ Ärzt_innen; siehe Kategorie l-b), Diskreditierung von Betroffenen und ihren Aussagen sowie die Sabotage von polizeilichen Ermittlungen.

„Und dann war diese Schwangerschaft, die man auch vertuschen wollte. Es war eine illegale Abtreibung, also eine Spätabtreibung. “

„Datenträger (mit Missbrauchsabbildungen von mir) verschwanden bei der Staatsanwaltschaft spurlos aus den Ermittlungsakten. “

\section{e) Täter_innen und das loyale Familiensystem}

Es wird beschrieben, dass Betroffene von ORG oft in Familienstrukturen hineingeboren würden, in denen mindestens ein Familienmitglied, oft aber mehrere, zu den aktiv Gewalt anwendenden Täter_innen gehörten und weitere, von den Gewalttaten wissende, Familienmitglieder passiv blieben. Beispielsweise 
> Tab. 3 Oberkategorie II: Betroffene Personen.

\section{Faktoren, die die Aufdeckung von organisier- ter und ritueller Gewalt erschweren (Unter- kategorien $a-d$ )}

Dissoziative Identitätsstörungen (DIS)

59

DIS-Anteil funktioniert im Alltag unauffällig (32)

DIS-Anteil diskreditiert Opfer(-aussagen) (19)

DIS-Anteil wird Mittäter_in (8)

dissoziative Amnesien

74

b) Hilflosigkeit

Angst vor Täter_innen 38

Mangel an Vertrauen 4

Erwartung von Unglaube 3

fehlende Kraft für Offenlegung

Erwartung von Machtlosigkeit

12

c) Schweigen

d) Abwehrmechanismen

Verdrängung

12

Verleugnung

Rationalisierung a) dissoziative Störungen

wird geschildert, dass Mütter ihre aus Vergewaltigungen entstandenen Kinder nicht schützten, weil sie diese nicht lieben können. Die teils als patriarchal-hierarchisch beschriebenen Familiensysteme isolierten sich nach außen, indem die Täter_innen den Familienmitgliedern Kontakt zu Menschen außerhalb dieses Systems unterbänden (z.B. Verbote, mit anderen Kindern zu spielen). Weiterhin komme es vor, dass die Familienmitglieder, inklusive betroffener Personen, bei einer Bedrohung des Familiensystems (z. B. Auftritt des Jugendamtes) aus Angst die Täter_innen schützten. Es wird berichtet, dass Betroffene von ORG häufig ungewollte oder für einen Kult geborene Kinder seien, die sich in einer emotionalen und existenziellen Abhängigkeit zu ihrer Familie befänden, über welche ein autoritärer Vater walte.

„Und über allem hing drohend das Schweigegebot: ,Was in unserer Familie geschieht, geht niemanden etwas an!' Dies war unser aller Normalität, von der sowohl wir Kinder als auch unsere Mutter betroffen war. Wir sprachen darüber weder miteinander noch mit anderen Menschen. Außerhalb unserer Familie wusste meines Wissens niemand etwas davon. [...] Freundschaften mit Gleichaltrigen waren nicht erwünscht und wurden soweit möglich unterbunden in meinem Fall verboten. [...] Dass ich extrem abgeschottet aufwuchs und nur sehr selten außerfamiliäre Kontakte zugelassen wurden, hat bestimmt auch Chancen verhindert, dass jemand auf meine Not aufmerksam werden konnte."

\section{Oberkategorie II: Betroffene Personen}

In $>$ Tab. 3 werden Unterkategorien dargestellt, in denen sich durch die betroffenen Personen beeinflusste Faktoren ausdrücken, die zur Verschleierung von Strukturen organisierter und ritueller Gewalt beitragen.

\section{a) Betroffene Personen und dissoziative Störungen}

Die in Kategorie I- $a$ beschriebene Gewalteinwirkung durch die Täter_innen kann bei den Betroffenen zu dissoziativen Störungen führen. Diese prägen sich laut der Berichte in Form von dissoziativen Amnesien und dissoziativen Identitätsstörungen aus, wobei diese psychischen Erkrankungen von den Täter_innen teils strategisch induziert und genutzt würden, um Kontrolle über die betroffenen Personen auszuüben. Beispielsweise wird geschildert, dass bestimmte Persönlichkeitsanteile der betroffenen Personen die Aufdeckung von ORG insofern behinderten, dass diese im Alltag unauffällig funktionierten (z. B. gute Schulnoten und Erfolg im Job). Andere Anteile wiederum seien täter_innen-loyal und würden Aussagen durch ein absichtsvoll unglaubwürdiges Verhalten diskreditieren. Darüber hinaus werden in den Berichten die Erfahrungen geschildert, dass Aussagen von Personen mit dissoziativen Störungen in der juristischen Wertung per se angezweifelt würden.

"Gerade weil die Taten so furchtbar sind und den Opfern so schreckliches Leid zugefügt wird, dass ihre Seele z. B. nur durch Dissoziation weiterleben kann, sind Täter bestens geschützt. Denn die Justiz/die Rechtsprechung sagt, Aussagen von Opfern mit dissoziativer Identitätsstörung sind im Strafverfahren untauglich. Fast absurd auch die Tatsache, dass für eine Anklageerhebung Betroffene im Strafverfahren detaillierte Auskünfte zu Wer? Was? Wo? Wann? Wie? liefern müssen. Auch hier haben organisierte Täterringe quasi schon von vornherein gewonnen."

„Die Täter kriegen einen Freibrief. Weil ich eine dissoziative Persönlichkeitsstörung habe, bin ich nicht glaubwürdig. Also wenn ich die Kinder ... Je mehr ich die spalte, desto unglaubwürdiger sind die, also kann der Täter machen, was er möchte. Weil es passiert ihm ja nichts. Die Staatsanwaltschaft sagt: Nein, das ist nicht glaubwürdig. Das wird abgeschmettert. “

Außerdem wird geschildert, dass Erinnerungen an die Gewalterfahrungen bei Betroffenen oft erst Jahre später und/ oder nur fragmentiert einsetzen und sie ihren Erinnerungen nach sogenannten dissoziativen Amnesien teilweise selbst nicht trauen würden.

„Wir wussten nicht, dass wir missbraucht worden sind. [...] Wir wussten nur, dass wir nichts wissen. Aber das hat uns jetzt nicht groß beunruhigt. Wir dachten, es ist normal, dass man sich an seine Kindheit nicht erinnert. [...] Das Erste, was kam, war die Konfirmation mit 14.Da hat die Erinnerung eingesetzt. “

\section{b) Betroffene Personen und ihre Hilflosigkeit}

Nach den Angaben der Berichtenden befinden sich die von ORG betroffenen Personen in einem Zustand der Hilflosigkeit, die auf einer starken, allgegenwärtigen (Todes-)Angst vor den Täter_innen und wiederholter Gewalt beruht. Von den Betroffenen wird außerdem ein Mangel an Vertrauen bis hin zu Misstrauen gegenüber anderen Menschen und einer Erwartung von 
Unglaube im Falle einer Offenlegung ihrer Gewalterfahrungen berichtet. Mit diesem Hintergrund wird erläutert, dass eine Offenlegung zu viel Kraft koste, als dass sie zu bewältigen sei. Weiterhin wird in den Berichten eine geringe Wirksamkeitserwartung beziehungsweise das Gefühl von Machtlosigkeit deutlich.

„Diese Angst, was passiert mit mir, wenn ich es jetzt wirklich öffentlich mache und vielleicht meine Eltern dann irgendwie vorgeladen werden zur Behörde oder zum Gericht, was passiert dann mit mir? Also bin ich wirklich meines Lebens dann noch sicher?"

„Weil, zum einen denken wir, dass ganz schnell Menschen sich abkehren würden, Schubladen aufgemacht und wieder zu, und man kommt nie wieder raus aus so einer Schublade. [...] Deswegen wollen wir auch nicht damit in die Öffentlichkeit, da sind wir ganz klar, das geht nicht. Und das ist natürlich ein Dilemma, weil, es muss eine Öffentlichkeit hergestellt werden. Und es wäre das Beste, wenn die Betroffenen in die Öffentlichkeit gehen würden. Aber für unser Leben ist es nicht realisierbar."

„Nicht beschreibbar, welch Kraftakt es jedes Mal ist, gegen dieses Gebot zu verstoßen und die grauenhaften Wahrheiten auszusprechen oder sogar aufzuschreiben. “

„Wie gesagt, mein Vater war Richter. Wer glaubt mir denn solche ungeheuerlichen, unaussprechlichen Geschichten?"

„Da habe ich keine Kraft für, da irgendwie so einen Krieg anzufangen."

\section{c) Betroffene Personen und ihr Schweigen}

Es wird in den Berichten deutlich, dass die betroffenen Personen, bedingt durch Angst und die Erwartung von Machtlosigkeit (siehe Kategorie II-b), annehmen, eine Offenlegung der Gewalterfahrungen würde nicht helfen und verschlimmere die Situation, weshalb Schweigen alternativlos sei.

„Und letztlich war es so, dass die gefragt haben, ob sie das Jugendamt einschalten sollen, weil, die wussten, irgendwas stimmt da nicht. Und wir haben nur mit völliger Panik reagiert. Bloß nicht! Also wir haben es abgelehnt und gesagt: Bitte, bitte nicht, das wird alles ganz schlimm und so. [...] Wir hatten so Angst. Wir waren ganz sicher, wir sterben, wenn wir reden."

„Warum sind denn diese Zirkel bisher so unangetastet? Warum gibt es nicht genauso Verfahren wie beim NSU in Bezug auf diese Zirkel? Weil die Opfer nicht sprechen.“

\section{d) Betroffene Personen und ihre Abwehrmechanismen}

Aus den Berichten lassen sich psychische Abwehrmechanismen erschließen, die als Bewältigungsprozesse in der psychischen Struktur der Betroffenen unbewusst dem Schutz des psychischen Gleichgewichts dienen können. Gleichzeitig tragen diese vermutlich dazu bei, die erlebten Gewalterfahrungen nicht bewusst werden zu lassen, was eine Offenlegung verhindern kann. Abwehrmechanismen, die besonders deutlich wurden, sind Verdrängung, Verleugnung und Rationalisierung. Der letzteren Kategorie wurden Textpassagen zugeordnet, in denen Betroffene sich selbst die Schuld für die Missbrauchserfahrungen gaben oder derartige Gewalt als Normalität rechtfertigten, die so auch in anderen Familien stattfinden würde.

„Es gibt bei mir auch das Phänomen, dass ich zwischendurch immer denke: Ist doch gar nichts passiert. Woher weiß ich das

Tab. 4 Oberkategorie III: Andere Personen (Umfeld, Gesellschaft, Justiz).

\section{Faktoren, die die Aufdeckung von organi- sierter und ritueller Gewalt erschweren (Unterkategorien a-d)}

\begin{tabular}{|c|c|c|}
\hline a) Ignoranz & & 115 \\
\hline fehlendes Konzept von ORG & 15 & \\
\hline Missachtung von Hinweisen & 21 & \\
\hline Mangel an Hilfestrukturen & 17 & \\
\hline Unglaube & 41 & \\
\hline ORG als Tabuthema & 3 & \\
\hline Passivität & 18 & \\
\hline b) strafrechtliche Hürden & & 42 \\
\hline $\begin{array}{l}\text { belastende/abschreckende Glaubhaftigkeits- } \\
\text { begutachtungen }\end{array}$ & 28 & \\
\hline $\begin{array}{l}\text { Dysfunktionalität aussagepsychologischer } \\
\text { Kriterien }\end{array}$ & 3 & \\
\hline strafrechtliche Verjährungsfrist & 11 & \\
\hline c) Abwehrmechanismen & & 40 \\
\hline Verdrängung & 5 & \\
\hline Verleugnung & 22 & \\
\hline Rationalisierung & 13 & \\
\hline d) Hilflosigkeit & & 9 \\
\hline Angst vor Täter_innen & 4 & \\
\hline Erwartung von Machtlosigkeit & 5 & \\
\hline
\end{tabular}

denn? Es wird so stark auch mit den eigenen Verdrängungsmechanismen operiert. Diese ganzen schlimmen Dinge, die gehen so über die Grenzen des eigenen Verstandes und die Grenzen des eigenen Fassungsvermögens raus, dass ich zwischendurch immer wieder so eine weiße Leinwand denke: War überhaupt irgendwas?"

„Wir sind da ja quasi aufgewachsen über Jahre, und das war die Normalität. Das war einfach ganz normal. Und dann irgendwann zu kapieren, dass es nicht normal ist, das war echt ein Weg. Also das klingt verrückt, aber das war tatsächlich ein Weg zu verstehen, dass es nicht die Normalität ist, dass es die Ausnahme ist, sozusagen."

\section{Oberkategorie III: Andere Personen}

In \ Tab. 4 werden Unterkategorien dargestellt, in denen durch andere Personen beeinflusste Faktoren erfasst werden, die zur Verschleierung von ORG-Strukturen beitragen. Die Gruppe anderer Personen setzt sich aus Menschen zusammen, die im direkten Kontakt zu Betroffenen stehen (z. B. Therapeut_innen, Lehrer_innen, Freunde, passive Familienmitglieder), aber auch aus Teilen der Gesellschaft (z. B. der Justiz). 
a) Das Umfeld, die Gesellschaft und Ignoranz

Im direkten Umfeld der Betroffenen sowie auf gesellschaftlicher Ebene wird eine vielschichtige Ignoranz (im Definitionssinne einer Missachtung durch Unkenntnis bis hin zu einer Missachtung trotz Kenntnis) gegenüber den betroffenen Personen sowie generell gegenüber dem Thema ORG berichtet. Diese Ignoranz könne dadurch zustande kommen, dass Menschen ein fehlendes Konzept von solchen Gewaltstrukturen hätten und durch ihr Unwissen darauf hindeutende Hinweise weniger wahrnehmen würden. Darüber hinaus ginge die beschriebene Ignoranz im direkten Umfeld der Betroffenen mit Skepsis hinsichtlich des Realitätsbezugs der offenbarten Gewalttaten einher. Auf gesellschaftlicher Ebene offenbare sich die Ignoranz gegenüber dem Thema ORG außerdem in einem Mangel an Hilfestrukturen, insbesondere im Bereich der psychosozialen Versorgung. Weiterhin lässt sich aus den Berichten schließen, dass ORG ein gesellschaftliches Tabuthema darstellt und dass Personen im direkten Umfeld der Betroffenen die Aufdeckung der Gewaltstrukturen erschweren, indem sie passiv bleiben oder betroffenen Personen davon abraten, das Schweigen zu brechen.

„Leider gibt es bis heute eigentlich kein brauchbares Netzwerk, was geeignete Hilfen für einen Ausstieg aus so einem Kult bietet! [...] Psychotherapie ist harte Arbeit und oftmals die einzige Möglichkeit für Betroffene, wenigstens eine Verbesserung ihrer Lebensund Leidenssituationen zu erlangen. Gerade für komplex traumatisierte Betroffene, ist die von den Krankenkassen übernommene Stundenzahl fast lächerlich gering. “

„Wir wollen da ja auch raus. Aber uns wird es so schwer gemacht, auch da rauszukommen. Nicht nur durch die Sekte, sondern auch durch die Gesellschaft."

„Ja, das Problem ist einfach, dass die Umwelt da überhaupt kein Auge dafür hat, was da so los ist in der Familie oder, dass nach auBen alles so gut getarnt ist, dass anscheinend alles in Ordnung ist."

„Und dass auch einfach das Thema rituelle Gewalt in die Gesellschaft kommt, dass auch Erzieher davon hören oder Lehrer. Weil, das ist einfach nicht bekannt, dass es sowas gibt bei uns. Neben unserer Tür sozusagen."

„Also ich könnte mir vorstellen, wenn mehr Leute aufgeklärt werden, mehr hingucken und vielleicht auch mal schneller was sagen. Dass das helfen könnte. “

\section{b) Die Justiz und strafrechtliche Hürden}

Es wurden strafrechtliche Hürden benannt, welche die Aufdeckung von ORG aus Sicht der Betroffenen erschweren. Beispielsweise würden Glaubhaftigkeitsbegutachtungen als belastend und abschreckend wahrgenommen. Weiterhin wird deutlich, dass aussagepsychologische Begutachtungen von den Berichtenden als problematisch erlebt werden, weil traumaassoziierte Gedächtnisstörungen (siehe Kategorie II- $a$ ), in Begutachtungen per se ein Merkmal für Unglaubwürdigkeit darstellten. Außerdem wird empfunden, dass die strafrechtliche Verjährungsfrist in Deutschland dazu beitrage, dass späte Strafanzeigen (die keine Tötung einschließen) wirkungslos blieben.

„Das ist ein Gutachterverfahren, da gehst du raus, da kannst du dich hinterher einweisen lassen, der nimmt dich so auseinander ..."
„Was sexuellen Kindesmissbrauch und organisierte sexuelle Gewalt angeht, schützt unser derzeitiges Rechtssystem vor allem die Täter. Insbesondere, was Tätergruppen auf dem Hintergrund organisierter/ritueller Gewalt angeht, ist meines Erachtens eine Strafverfolgung dieser Gruppen gemäß derzeitiger Rechtsprechung nahezu ausgeschlossen."

„Verjährung. Auch diese dient dem Schutz der Täter. Der Weg raus aus organisierten Kulten ist oft ein jahrzehntelanger. Auch ist inzwischen hinreichend bekannt, dass Betroffene oft viele, viele Jahre brauchen, um über ihre Erlebnisse zu sprechen. Haben Opfer dann so weit Abstand, um vielleicht tatsächlich in einem Strafverfahren aussagefähig zu sein, sind die Taten oft längst verjährt.“

\section{c) Das Umfeld, die Gesellschaft und ihre} Abwehrmechanismen

Abwehrmechanismen wurden nicht nur bei den von ORG betroffenen Personen (siehe Kategorie II-d), sondern auch bei Personen in deren direktem Umfeld und in Teilen der Gesellschaft deutlich. Aus den Berichten ließen sich ebenfalls Verdrängung, Verleugnung und Rationalisierung erschließen.

„Und dann wollte ich halt nicht zum Arzt, und das Ergebnis war: Ja, wenn du nicht zum Arzt gehst, gab es keine Vergewaltigung. Und das war es. “

„Das ist ja eine ganz klare Aussage darüber, wo stehen wir gesellschaftlich an dem Punkt. Das darf nicht sein, ja? Das wird also auch gesellschaftlich komplett verdrängt. “

"Sexuelle Gewalt oder gar organisierte/rituelle Gewalt ist heute noch immer ein großes Tabuthema und war es damals umso mehr. Diese Wirklichkeit, die schlimmste sexuelle Ausbeutung von Kindern inmitten unserer so heilen Gesellschaft, will niemand hören oder gar glauben. Manchmal denke ich, die Täter machen eigentlich alles, richtig: Denn je schlimmer und grausamer die Taten, umso unwahrscheinlicher eine Entdeckung und Strafverfolgung weil ,nicht sein kann, was nicht sein darf“."

„Ich glaube, was ich von der Gesellschaft erwarte, ist, erst mal alles für möglich zu halten. Also nicht sagen: Das kann nicht sein, das gibt es nicht. Das ist das, was ich als wichtig ansehe."

\section{d) Das Umfeld und Hilflosigkeit}

Hilflosigkeit in Form von Angst und Machtlosigkeit wurde nicht nur bei den von ORG betroffenen Personen (siehe Kategorie IIb), sondern auch in deren direktem Umfeld deutlich, wie das folgende Zitat einer psychosozialen Fachperson zeigt, die von ORG Betroffene professionell begleitet hat:

„ICh wäre diskreditiert gewesen, wenn wir das gemacht hätten, ne? Die hätten uns fertiggemacht mit so was. Dann hätte ich da irgend so ein hohes Tier angeklagt. “

\section{Diskussion}

Mittels einer qualitativen Inhaltsanalyse von Betroffenen- und Zeitzeug_innen-Berichten wurden in der vorliegen Arbeit Faktoren identifiziert, die das Aufdecken von ORG in Deutschland erschweren. In der Analyse bildeten sich 3 Personengruppen ab (Oberkategorien), die auf verschiedenen Ebenen (Unterkategorien) wechselseitig und teils zirkulär miteinander interagieren. In diesem Interaktionsprozess scheinen sich in Bezug auf 
die Fragestellung strukturelle Bedingungsfaktoren für eine erschwerte Aufdeckung von ORG-Strukturen zu etablieren, deren Zusammenhänge im Folgenden diskutiert werden.

Den Berichten zufolge stellen die Täter_innen eine aktive Personengruppe im Prozess der Verschleierung von ORG dar, die durch Anwendung psychischer, körperlicher und sexualisierter Gewalt (z. B. Erpressung, Folter und Zwangsprostitution) Kontrolle über direkt betroffene Personen, die Betroffenen unterstützende Personen und erpressbare Fremdtäter_innen erlangen. Aus den Ergebnissen kann geschlossen werden, dass ein Machtgefälle zwischen den Täter_innen und betroffenen Personen besteht, wobei Hilflosigkeit bei den Betroffenen Abwehrmechanismen und dissoziative Symptome bahnen kann, die dazu beitragen, solch extreme Gewalterfahrungen psychisch bewältigen zu können [11].

Der Bericht von Erpressung durch erzwungene Wechsel der Opfer- in Täter_innenrollen deckt sich mit den aus einer anonymen Onlinebefragung gewonnenen Ergebnissen [8] und dient vermutlich dem Zweck, bestehende Abhängigkeitsverhältnisse zu verstärken [1]. Darüber hinaus kann die Hilflosigkeit durch ein generalisiertes Misstrauen der Betroffenen gegenüber Menschen verstärkt werden, indem Täter_innen suggerieren, es sei von außen keine Hilfe zu erwarten, da Personen aus der Ärzteschaft, Polizei und Strafverfolgung mit zur Täter_innengruppe gehören [1]. Das aus diesen Bedingungsfaktoren resultierende Schweigen betroffener Personen ist nicht nur typisch für ORGKontexte, sondern bildet sich auf ähnliche Weise in verschiedensten Kontexten sexuellen Missbrauchs ab [26]. Vor diesem Hintergrund ist eine Aufarbeitung der Geschehnisse elementar, deren kommunikativer Prozess auf dem Aussprechen und Anhören von Wahrheiten basiert [1].

Bei mitwissenden Personen im direkten Umfeld der Betroffenen resultiert die dort ebenso deutliche Hilflosigkeit neben ähnlichen psychischen Abwehrmechanismen auch in einer Ignoranz gegenüber verschiedenen Aspekten von ORG. Diese kann sich, den Ergebnissen zufolge, zum Beispiel in einer naiven Missachtung von Gewaltspuren, in Ungläubigkeit gegenüber Betroffenenberichten oder in einer gesellschaftlichen Tabuisierung des Themas äußern. Dabei würde nach Einschätzung der UKASK verkannt, dass eine solche Einstellung der Sachlage nicht gerecht werde, da das Nutzbarmachen einer Weltanschauung oder Religion zur Rechtfertigung der Auslebung von sexuellen Gewaltfantasien aufgrund vieler aktuell sichtbar gewordener Missbrauchsfälle nicht mehr außerhalb der Vorstellungskraft liegen dürfe und sich die Erfahrungsberichte von Betroffenen und psychosozialen Fachpersonen nicht mehr leugnen ließen [1]. Um psychischer Abwehr und Ignoranz im direkten Umfeld der Betroffenen sowie in der Gesellschaft entgegenzuwirken, ist unserer Meinung nach das Generieren und transparente Vermitteln von Wissen notwendig, um einen offeneren und respektvolleren Diskurs zu fördern und der bisher oft üblichen Polarisierung bzw. Spaltung im Themenbereich ORG entgegenzuwirken. In diesem Zusammenhang kann es von entscheidender Bedeutung sein, Informationen zum Thema ORG sachlich-objektiv und möglichst nicht skandalisierend aufzubereiten und inhomogen verwendete und dadurch teilweise fehlleitende Begriffe, wie „rituell“, klar zu definieren und einzuord- nen [8]. Weiterhin ist es nach dem Forderungskatalog des UBSKM notwendig, die Zusammenarbeit von Wissenschaft und Praxis zu verbessern, Betroffene mehr an Forschungsprojekten zu beteiligen und Forschungsergebnisse zu sexuellem Missbrauch besser in die Aus- und Weiterbildung von Fachkräften zu integrieren, die mit Kindern und Jugendlichen arbeiten [27].

Ferner weisen die Ergebnisse darauf hin, dass eine gute gesellschaftliche Positionierung der Täter_innen diese davor schützen kann, als solche wahrgenommen bzw. zur Rechenschaft gezogen zu werden. Insbesondere durch eine Vernetzung von Täter_innengruppen und durch finanzielle Ressourcen, die durch Zwangsprostitution oder die Herstellung sogenannter Kinderpornografie gewonnen werden, können Täter_innen scheinbar ihre Macht stabilisieren. Auch die UKASK nimmt an, dass sexueller Kindesmissbrauch in organisierten Gruppen häufig kommerziellen Motiven folge [1]. Der Bericht einer transgenerationalen familiären Einbindung in die Gewaltstrukturen deckt sich mit Ergebnissen aus Fallstudien und einer anonymen Onlinestudie $[4,28]$. Die aktiven und passiven Täter_innen sowie die betroffenen Personen scheinen dabei teilweise täter_innen-loyale Familiensysteme zu bilden, die sich isolieren und bei Angriffen von außen die Täter_innen schützen, indem Gewalttaten verschwiegen oder vertuscht werden.

Mit den Ausführungen des Fachkreises für sexualisierte Gewalt in ORG-Strukturen beim BMFSFJ deckt sich das Ergebnis, dass die Täter_innen ihre Kontrolle über die betroffenen Personen schon im frühen Kindesalter durch Manipulation zu bahnen und auszubauen scheinen [3]. Die vorliegenden Ergebnisse legen neben der Induktion von DIS, Programmierung dissoziativer Anteile, Gaslighting und ideologischer Indoktrination nahe, dass Täter_innen kindliche Grundbedürfnisse in einem Machtgefälle ausnutzen, um deren Zugehörigkeitsgefühl zur Täter_innengruppe zu sichern. Zur Vertuschung von Gewalttaten ergriffen Täter_innen aktive Maßnahmen, wie durch Erpressung forcierte Schweigegebote oder gezielte Täuschung über Tatorte, beteiligte Personen und Geschehensabläufe. Die Diskreditierung von Erlebnisberichten der betroffenen Personen zum Zweck der Vertuschung erfordere kaum noch ein Zutun der Täter_innen, da die Drastik der berichteten Gewalt bei anderen Personen (aber auch bei den Betroffenen selbst) schnell zu Abwehr und Unglaube führe und dissoziative Störungen der betroffenen Personen in der Wahrnehmung anderer Personen die Glaubwürdigkeit der Betroffenen per se mindern würden. Die Möglichkeit, dass Täter_innen bestimmte Persönlichkeitsanteile der betroffenen Personen kontrollieren, kann für die Betroffenen selbst, aber auch für unterstützende Fachpersonen in der psychosozialen Versorgung [29, 30] und der Justiz [31, 32] eine schwer berechenbare Herausforderung darstellen. Darüber hinaus ist die Diagnose einer DIS in der alltäglichen klinischen Praxis oft unbekannt oder nicht anerkannt [10], was nach Angaben der von ORG betroffenen Personen zu häufigen Fehldiagnosen führt [4] und zur mangelhaften Versorgungssituation $[5,16]$ beiträgt. Um diesen Herausforderungen zu begegnen, sind professionelle Informations- und Anlaufstellen für eine geschützte Ausstiegsbegleitung, eine dauerhafte finanzielle Unterstützung der Fachberatungsstellen und Opferver- 
bände, bedarfsgerechte und qualifizierte Therapiemöglichkeiten sowie eine Reform des Opferentschädigungsrechts nötig [1].

Aufgrund von strafrechtlichen Hürden aus der Perspektive der von ORG betroffenen Personen bleibt eine Verfolgung der Gewalttaten in den ohnehin wenigen angezeigten Fällen scheinbar oft aus, was die Aufdeckung von ORG-Strukturen erschweren kann. Neben abschreckend belastenden Begutachtungsprozessen sei es den Betroffenen aufgrund von Vertuschungsstrategien der Täter_innen kaum möglich, ausreichende Tatnachweise zu führen, da meistens Zeug_innen fehlten. Darüber hinaus scheiterten Personen mit Traumafolgestörungen häufig an den in Deutschland gängigen Kriterien der aussagepsychologischen Glaubhaftigkeitsbegutachtung. Sobald Betroffene von ORG sich dem Justizsystem stellten, durchlebten sie oft dasselbe wie in Momenten, in denen sie sich Personen im direkten Umfeld anvertraut hätten: ihnen werde nicht geglaubt und sie seien machtlos. Deshalb sollte dieses Verfahren auf die Anwendung unter solch besonderen Begutachtungsumständen überprüft und angepasst werden [11, 33].

\section{Limitationen}

Bei der Interpretation der Ergebnisse dieser Studie soll hervorgehoben werden, dass das Auswahlverfahren der „anfallenden Stichprobe“ keine speziellen Auswahlkriterien anlegt und somit weder besonders informationshaltige Fälle gewinnt, noch Fälle, die Repräsentativität für die Grundgesamtheit beanspruchen könnten. Der Hintergrund des untersuchten Datenmaterials impliziert außerdem, dass die bearbeitete Fragestellung den Berichtenden nicht direkt vorgegeben wurde, sondern, dass die Beantwortung auf der Interpretation vorhandener und als relevant bewerteter Textpassagen durch die Autor_innen basiert. Eine weitere Limitation ist die eingeschränkte Möglichkeit zur Veröffentlichung direkter Zitate, welche sich durch den besonderen Partizipations- und Datenschutzfokus der Betroffenen und Zeitzeug_innen im gegebenen Kontext erklärt und gleichzeitig die Augenscheinvalidität des Kategoriensystems begrenzt.

\section{Schlussfolgerung}

Die Ergebnisse dieser Studie legen nahe, dass neben den bezeichneten Täter_innen und betroffenen Personen auch andere Personen (im direkten Umfeld sowie als Teil der Gesellschaft) in systemischen Interaktionsprozessen Bedingungen schaffen können, welche die Aufdeckung von ORG in Deutschland erschweren. Um dieses System zu verändern, ist eine sachliche und sensible Aufklärungsarbeit an verschiedenen gesellschaftlichen Institutionen hinsichtlich der Wahrnehmung, Eindämmung und Bewältigung typischer Herausforderungen im Bereich der ORG notwendig. Besonders hervorzuheben sind hierbei die vielschichtigen Machtverhältnisse zwischen Täter_innen und betroffenen/anderen Personen, verschwimmende Grenzen zwischen Opfer- und Täterschaft, Manipulations- und Vertuschungsstrategien, dissoziative Störungen bei den Betroffenen und eine unspezifische, aber gängige Kultur des Schweigens. Eine transparent informierte Gesellschaft, zum Beispiel durch professionelle Schulungen in pädagogischen Einrichtungen, in der Gesundheitsversorgung, in der Justiz und bei der Polizei, könnte dazu beitragen, ORG-Strukturen stärker entgegenzuwirken und Betroffene besser zu unterstützen.

KONSEQUENZEN FÜR KLINIK UND PRAXIS

- Mit Patient_innen, die im klinischen Kontext sexuellen Kindesmissbrauch in organisierten und rituellen Gewaltstrukturen berichten, sollte ein adäquater und sensibler Umgang gefunden werden.

- Klinische Fachpersonen sollten dabei unterstützt werden, ein informierteres Konzept von organisierter und ritueller Gewalt sowie von dissoziativen Identitätsstörungen zu entwickeln.

- Es ist notwendig, professionelle Beratungs- und Anlaufstellen für eine geschützte Ausstiegsbegleitung zu subventionieren.

\section{Danksagung}

Die Autor_innen danken allen Betroffenen und Zeitzeug_innen im Kontext organisierter und ritueller Gewalt, die der UKASK ihre Berichte oder Anhörungen auch für die Forschung zur Verfügung gestellt haben. Außerdem vielen Dank an Ricarda Bauch, Jasmin Michaelis, Sascha Dobbrunz und Elisabeth Stück für ihre Unterstützung.

\section{Funding}

Die vorliegende Studie wurde durch die Unabhängige Kommission zur Aufarbeitung sexuellen Kindesmissbrauchs (UKASK) gefördert, die vom Unabhängigen Beauftragten für Fragen des sexuellen Kindesmissbrauchs (UBSKM) ernannt wurde. Das Amt des UBSKM ist mit dem Bundesministerium für Familie, Senioren, Frauen und Jugend (BMFSFJ) assoziiert, arbeitet jedoch unabhängig vom BMFSFJ und ist nicht weisungsgebunden.

Interessenkonflikt

Peer Briken ist Mitglied der UKASK. Die Autoren/Autorinnen geben an, dass kein Interessenkonflikt besteht.

Literatur

[1] Unabhängige Kommission zur Aufarbeitung sexuellen Kindesmissbrauchs. Bilanzbericht 2019. Berlin: 2019

[2] Salter M. Organized abuse in adulthood: Survivor and professional perspectives. J Trauma Dissociation 2017; 18: 441-453

[3] Fachkreis „Sexualisierte Gewalt in organisierten und rituellen Gewaltstrukturen“ beim BMFSFJ. Sexualisierte Gewalt in organisierten und rituellen Gewaltstrukturen - Prävention, Intervention und Hilfe für Betroffene stärken - Empfehlungen an Politik und Gesellschaft. 2018: 1-36

[4] Schröder J, Nick S, Richter-Appelt $\mathrm{H}$ et al. Psychiatric impact of organized and ritual child sexual abuse: Cross-sectional findings from 
individuals who report being victimized. Int J Environ Res Public Health 2018; 15: e2417

[5] Nick S, Schröder J, Briken P et al. Organisierte und rituelle Gewalt in Deutschland - Kontexte der Gewalterfahrungen, psychische Folgen und Versorgungssituation. Trauma \& Gewalt 2018; 12: 1-19

[6] Salter M. The Role of Ritual in the Organised Abuse of Children. Child Abus Rev 2012; 21: 440-451

[7] Behrendt P, Schröder J, Nick S et al. Was ist sexualisierte Gewalt in organisierten und rituellen Gewaltstrukturen? Eine qualitative Inhaltsanalyse der Erfahrungsberichte von Betroffenen. Zeitschrift für Sexualforschung, in Revision

[8] Schröder J, Nick S, Richter-Appelt $\mathrm{H}$ et al. Demystifying the 'ritual' in organized child sexual abuse - Insights of self-identified victims and health care professionals. J Trauma Dissociation 2020: doi:https:// doi.org/10.1080/15299732.2020.1719260

[9] Kownatzki R, Eilhardt S, Hahn B et al. Rituelle Gewalt - Umfragestudie zur satanistischen rituellen Gewalt als therapeutisches Problem. Psychother aktuell 2012; 57: 70-76

[10] Gast U, Rodewald F, Hofmann A et al. Die dissoziative Identitätsstörung - häufig fehldiagnostiziert. Dtsch Arztebl 2006; 103: 31933200

[11] Igney C. Rituelle Gewalt - im Spannungsfeld von Parallelwelten, gesellschaftlicher (Ab-)Spaltung und psychosozialem Arbeitsalltag. Zeitschrift für Psychotraumatologie, Psychother Psychol Medizin 2012; $12: 11-26$

[12] Hehl F-J, Werkle R. Eine retrospektive Untersuchung von familiären Beziehungsstrukturen bei sexuellem Mißbrauch - eine Pilotstudie. Zeitschrift für Fam 1993; 5: 215-248

[13] Rodewald F, Wilhelm-Gößling C, Emrich HM et al. Axis-I comorbidity in female patients with dissociative identity disorder and dissociative identity disorder not otherwise specified. J Nerv Ment Dis 2011; 199: 122-131

[14] Farham B. Sexual violence against children leads to serious health problems. South African Med J 2018; 108: 361-369

[15] Young WC, Sachs RG, Braun BG et al. Patients reporting ritual abuse in childhood: A clinical syndrome: Report of 37 cases. Child Abuse Negl 1991; 15: 181-189

[16] Sommer J. Die psychotherapeutische Versorgungsrealität komplex traumatisierter Menschen in Deutschland. Trauma \& Gewalt 2016; 10: 308-319

[17] Ustupska MM, Stopsack M, Preibsch A et al. Rituelle Gewalt - ein blinder Fleck? Bewusstsein über Gewalt an Kindern und Jugendlichen bei Fachkräften im Sozial- und Gesundheitswesen. Trauma 2016; 14: $12-23$

[18] Salter M. Finding a new narrative: Meaningful responses to 'false memory' disinformation. In: Sinason V, ed. Memory in Dispute. London: Karnac; 2018: 1-10
[19] Betroffenenrat zum Umgang mit Ritueller Gewalt beim UBSKM. Die unendliche Geschichte: Rituelle Gewalt und die Unfähigkeit, den Betroffenen zu glauben. 2018: Im Internet: https://beauftragter-missbrauch.de/betroffenenrat/aktuelles/detail/statement-des-betroffenenrates-zum-umgang-mit-ritueller-gewalt

[20] Schreier M. Qualitative Stichprobenkonzepte. In: Naderer G, Balzer E, Hrsg. Qualitative Marktforschung in Theorie und Praxis. Wiesbaden: Gabler; 2007: 231-245

[21] Hwang S. Utilizing qualitative data analysis software: A review of Atlas.ti. Soc Sci Comput Rev 2008; 26: 519-527

[22] Mayring P. Qualitative Inhaltsanalyse. Grundlagen und Techniken. Weinheim: Beltz; 2003

[23] Hsieh H, Shannon S. Three approaches to qualitative content analysis. Qual Health Res 2005; 15: 1277-1288

[24] Gamer M, Lemon J, Fellows I et al. irr: Various Coefficients of Interrater Reliability and Agreement. R package version 0.84.1. 2019: Im Internet: https://cran.r-project.org/package=irr

[25] R Core Team. R: A language and environment for statistical computing. 2017: Im Internet: https://www.r-project.org

[26] Andresen S. Das Schweigen brechen. Kindesmissbrauch - Voraussetzungen für eine persönliche, öffentliche und wissenschaftliche Aufarbeitung. In: Geiss M, Magyar-Haas V, Hrsg. Zum Schweigen. Macht/ Ohnmacht in Erziehung und Bildung. Weilerswist: Velbrück Wissenschaft; 2015

[27] Unabhängiger Beauftragter für Fragen des sexuellen Kindesmissbrauchs. Forderungskatalog: Forschung zu sexuellem Missbrauch Vom Tabu zur gesamtgesellschaftlichen Aufgabe. 2015: Im Internet: https://beauftragter-missbrauch.de/fileadmin/Content/pdf/Pressemitteilungen/Forderungskatalog_Forschung.pdf

[28] Sarson J, MacDonald L. Ritual abuse-torture within families/groups. J Aggress Maltreatment Trauma 2008; 16: 419-438

[29] Nick S, Schröder J, Briken P et al. Organisierte und rituelle Gewalt in Deutschland: Praxiserfahrung, Belastungen und Bedarfe von psychosozialen Fachkräften. Trauma \& Gewalt 2019; 13: 114-127

[30] Nassan-Agha-Schroll H, Steidl M, Aigner M. Dissoziative Störung einst und jetzt - Ein Fallbericht. Zeitschrift für Prakt Psychiatr und Neurol 2020; 23: 24-27

[31] Paris ]. Dissociative identity disorder: Validity and use in the criminal justice system. BJPsych Adv 2019; 25: 1-7

[32] Farrell HM. Dissociative Identity Disorder: Mediocolegal Challenges. J Am Acad Psychiatry Law 2011; 39: 402-406

[33] Schoon W, Briken P. Zur Anwendbarkeit der Glaubhaftigkeitsbegutachtung unter spezifischen Begutachtungsumständen - Eine narrative Übersichtsarbeit. Forensische Psychiatr Psychol Kriminologie 2019; 13: 125-135 